PROCEEDINGS OF THE

AMERICAN MATHEMATICAL SOCIETY

Volume 133, Number 4, Pages 987-993

S 0002-9939(04)07779-2

Article electronically published on November 19, 2004

\title{
HILBERT-SAMUEL COEFFICIENTS AND POSTULATION NUMBERS OF GRADED COMPONENTS OF CERTAIN LOCAL COHOMOLOGY MODULES
}

\author{
M. BRODMANN AND F. ROHRER
}

(Communicated by Bernd Ulrich)

\begin{abstract}
Let $R=\bigoplus_{n>0} R_{n}$ be a Noetherian homogeneous ring with onedimensional local base ring $\left(R_{0}, \mathfrak{m}_{0}\right)$. Let $\mathfrak{q}_{0} \subseteq R_{0}$ be an $\mathfrak{m}_{0}$-primary ideal, let $M$ be a finitely generated graded $R$-module and let $i \in \mathbb{N}_{0}$. Let $H_{R_{+}}^{i}(M)$ denote the $i$-th local cohomology module of $M$ with respect to the irrelevant ideal $R_{+}:=\bigoplus_{n>0} R_{n}$ of $R$. We show that the first Hilbert-Samuel coefficient $e_{1}\left(\mathfrak{q}_{0}, H_{R_{+}}^{i}(M)_{n}\right)$ of the $n$-th graded component of $H_{R_{+}}^{i}(M)$ with respect to $\mathfrak{q}_{0}$ is antipolynomial of degree $<i$ in $n$. In addition, we prove that the postulation numbers of the components $H_{R_{+}}^{i}(M)_{n}$ with respect to $\mathfrak{q}_{0}$ have a common upper bound.
\end{abstract}

\section{INTRODUCTION}

Let $R=\bigoplus_{n \geq 0} R_{n}$ be a Noetherian homogeneous ring, so that $R$ is $\mathbb{N}_{0}$-graded with Noetherian base ring $R_{0}$ and of the form $R=R_{0}\left[\ell_{0}, \cdots, \ell_{r}\right]$ with finitely many elements $\ell_{0}, \cdots, \ell_{r} \in R_{1}$. Let $R_{+}:=\bigoplus_{n>0} R_{n}$ denote the irrelevant ideal of $R$. Moreover let $M$ denote a finitely generated graded $R$-module. For $i \in \mathbb{N}_{0}$ let $H_{R_{+}}^{i}(M)=\bigoplus_{n \in \mathbb{Z}} H_{R_{+}}^{i}(M)_{n}$ denote the $i$-th local cohomology module of $M$ with respect to $R_{+}$. Keep in mind that the $n$-th graded component $H_{R_{+}}^{i}(M)_{n}$ of $H_{R_{+}}^{i}(M)$ is a finitely generated $R_{0}$-module for all $n \in \mathbb{Z}$ and vanishes for all $n \gg 0$.

Motivated by the "tameness-problem" for coherent sheaves over projective schemes (cf. $[\mathrm{B}-\mathrm{H}]$ ) one is led to ask for the "asymptotic behaviour" of the graded components $H_{R_{+}}^{i}(M)_{n}$ for $n \rightarrow-\infty$. Namely, the mentioned tameness-problem is equivalent to the question of whether for each $i \in \mathbb{N}_{0}$ either $H_{R_{+}}^{i}(M)_{n}=0$ for all $n \ll 0$ or $H_{R_{+}}^{i}(M)_{n} \neq 0$ for all $n \ll 0$. We do not know any example in which this tameness property is not satisfied. On the other hand, tameness has been established until now in fairly special cases only (cf. [L, B-F-L]).

A more specific question is, whether for each $i \in \mathbb{N}_{0}$, the set $\operatorname{Ass}_{R_{0}}\left(H_{R_{+}}^{i}(M)_{n}\right)$ of associated primes of the $R_{0}$-module $H_{R_{+}}^{i}(M)_{n}$ is asymptotically stable for $n \rightarrow$ $-\infty$. This is true, for example, if $R_{0}$ is semilocal and either of dimension one or of dimension two and essentially of finite type over a field (cf. [B-F-L]). On the other

Received by the editors December 1, 2003

2000 Mathematics Subject Classification. Primary 13D45, 13 E10.

Key words and phrases. Local cohomology modules, graded components, Hilbert-Samuel polynomials. 
hand this "asymptotic stability of associated primes" need not hold in general, as can be deduced from examples of Singh [S] (cf. [B-K-S]) or Katzman [K] (cf. B-F-T] . It may fail, for example, if $R_{0}$ is regular local and of dimension four.

Finally, to be even more specific, one could ask whether certain numerical invariants of the $R_{0}$-modules $H_{R_{+}}^{i}(M)_{n}$ "behave well" if $i$ is fixed and $n$ goes to $-\infty$. To be more precise we say that a numerical function $f: \mathbb{Z} \rightarrow \mathbb{Z}$ is antipolynomial (of degree $<i$ ) if there is some polynomial $p \in \mathbb{Q}[\mathbf{x}]$ (of degree $<i$ ) such that $f(n)=p(n)$ for all $n \ll 0$. Moreover, if $\left(R_{0}, \mathfrak{m}_{0}\right)$ is local and $\mathfrak{q}_{0} \subseteq R_{0}$ is an $\mathfrak{m}_{0}$-primary ideal, let $e_{0}\left(\mathfrak{q}_{0}, T\right)$ denote the Hilbert-Samuel multiplicity of the finitely generated $R_{0}$-module $T$ with respect to $\mathfrak{q}_{0}$. Then, in this terminology we can say (cf. B-F-T ):

(1.1) If $\left(R_{0}, \mathfrak{m}_{0}\right)$ is local of dimension one, $\mathfrak{q}_{0} \subseteq R_{0}$ is $\mathfrak{m}_{0}$-primary and $i \in \mathbb{N}_{0}$, the functions given by

$$
\begin{aligned}
n & \mapsto \operatorname{length}_{R_{0}}\left(\Gamma_{\mathfrak{m}_{0}}\left(H_{R_{+}}^{i}(M)_{n}\right)\right), n \mapsto \operatorname{length}_{R_{0}}\left(0_{H_{R_{+}}^{i}} \underset{(M)_{n}}{:} \mathfrak{q}_{0}\right), \\
n & \mapsto \operatorname{length}_{R_{0}}\left(H_{R_{+}}^{i}(M)_{n} / \mathfrak{q}_{0} H_{R_{+}}^{i}(M)_{n}\right) \text { and } n \mapsto e_{0}\left(\mathfrak{q}_{0}, H_{R_{+}}^{i}(M)_{n}\right)
\end{aligned}
$$

are all antipolynomial of degree $<i$.

The first aim of this note is to show that under the above hypotheses a further numerical invariant of the $R_{0}$-modules $H_{R_{+}}^{i}(M)_{n}$ is antipolynomial of degree $<i$. Namely, assume that $\left(R_{0}, \mathfrak{m}_{0}\right)$ is local of dimension one, let $\mathfrak{q}_{0} \subseteq R_{0}$ be an $\mathfrak{m}_{0^{-}}$ primary ideal and fix $i \in \mathbb{N}_{0}$. We then have "asymptotic stability of associated primes" and so $\operatorname{dim}_{R_{0}}\left(H_{R_{+}}^{i}(M)_{n}\right)$ takes a constant value $\leq 1$ if $n \rightarrow-\infty$. Assume that this constant value is 1 . Then, for all $n \ll 0$ the Hilbert-Samuel polynomial of $H_{R_{+}}^{i}(M)_{n}$ with respect to $\mathfrak{q}_{0}$ can be written as

$$
P_{H_{R_{+}}^{i}(M)_{n}, \mathfrak{q}_{0}}(\mathbf{x})=e_{0}\left(\mathfrak{q}_{0}, H_{R_{+}}^{i}(M)_{n}\right)(\mathbf{x}+1)-e_{1}\left(\mathfrak{q}_{0}, H_{R_{+}}^{i}(M)_{n}\right),
$$

where $e_{1}\left(\mathfrak{q}_{0}, H_{R_{+}}^{i}(M)_{n}\right)=-P_{H_{R_{+}}^{i}}(M)_{n}, \mathfrak{q}_{0}(-1) \in \mathbb{Z}$ is the so-called first HilbertSamuel coefficient of $H_{R_{+}}^{i}(M)_{n}$ with respect to $\mathfrak{q}_{0}$.

We shall prove that the function given by $n \mapsto e_{1}\left(\mathfrak{q}_{0}, H_{R_{+}}^{i}(M)_{n}\right)$ is antipolynomial of degree $<i$ (cf. Theorem 3.1).

In addition we show that the postulation numbers of the $R_{0}$-modules $H_{R_{+}}^{i}(M)_{n}$ with respect to $\mathfrak{q}_{0}$, hence the numbers

$$
\begin{array}{r}
\mu\left(\mathfrak{q}_{0}, H_{R_{+}}^{i}(M)_{n}\right):=\inf \left\{r \in \mathbb{N}_{0} \mid \operatorname{length}_{R_{0}}\left(H_{R_{+}}^{i}(M)_{n} / \mathfrak{q}_{0}^{t+1} H_{R_{+}}^{i}(M)_{n}\right)\right. \\
\left.=P_{H_{R_{+}}^{i}}(M)_{n}, \mathfrak{q}_{0}(t), \forall t \geq r\right\}
\end{array}
$$

have a common upper bound for all $n \in \mathbb{Z}$ (cf. Theorem 3.3 ).

As for the unexplained terminology we refer to $[\mathrm{B}-\mathrm{S}]$ and to $[\mathrm{E}]$.

\section{Hilbert-Samuel COEfFicients in Dimension 1}

Throughout this section let $(A, \mathfrak{m})$ be a local Noetherian ring of dimension one, let $\mathfrak{q} \subseteq A$ be an $\mathfrak{m}$-primary ideal and let $T$ be a finitely generated $A$-module of dimension one. 
2.1. Notation and Remark. A) Let $P_{T, \mathfrak{q}}(\mathbf{x}) \in \mathbb{Q}[\mathbf{x}]$ denote the Hilbert-Samuel polynomial of $T$ with respect to $\mathfrak{q}$, so that

$$
P_{T, \mathfrak{q}}(n)=\operatorname{length}_{A}\left(T / \mathfrak{q}^{n+1} T\right) \text { for all } n \gg 0 .
$$

As $\operatorname{dim}(T)=1, P_{T, \mathfrak{q}}(\mathbf{x})$ is of degree 1 and we may write

$$
P_{T, \mathfrak{q}}(\mathbf{x})=e_{0}(\mathfrak{q}, T)(\mathbf{x}+1)-e_{1}(\mathfrak{q}, T)
$$

where $e_{0}(\mathfrak{q}, T) \in \mathbb{N}$ is the (Hilbert-Samuel) multiplicity of $T$ with respect to $\mathfrak{q}$ and $e_{1}(\mathfrak{q}, T) \in \mathbb{Z}$ is the first Hilbert-Samuel coefficient of $T$ with respect to $\mathfrak{q}$.

B) We set

$$
\bar{T}:=T / \Gamma_{\mathfrak{m}}(T),
$$

where $\Gamma_{\mathfrak{m}}(T)=\bigcup_{n \in \mathbb{N}}\left(0: \mathfrak{m}^{n}\right)$ denotes the $\mathfrak{m}$-torsion of $T$. Keep in mind that $\operatorname{Ass}_{A}(\bar{T})=\operatorname{Ass}_{A}(T) \backslash\{\mathfrak{m}\}$, so that $\operatorname{dim}(\bar{T})=\operatorname{depth}_{A}(\bar{T})=1$.

C) Keep the above hypotheses and notation. Then, for each $n \in \mathbb{N}_{0}$, there is a short exact sequence

$$
0 \rightarrow \Gamma_{\mathfrak{m}}(T) /\left(\Gamma_{\mathfrak{m}}(T) \cap \mathfrak{q}^{n} T\right) \rightarrow T / \mathfrak{q}^{n} T \rightarrow \bar{T} / \mathfrak{q}^{n} \bar{T} \rightarrow 0 .
$$

In particular,

$\operatorname{length}_{A}\left(T / \mathfrak{q}^{n} T\right)=\operatorname{length}_{A}\left(\bar{T} / \mathfrak{q}^{n} \bar{T}\right)+\operatorname{length}_{A}\left(\Gamma_{\mathfrak{m}}(A)\right)-\operatorname{length}_{A}\left(\Gamma_{\mathfrak{m}}(T) \cap \mathfrak{q}^{n} T\right)$.

By Artin-Rees there is some $n_{0} \in \mathbb{N}$ such that $\Gamma_{\mathfrak{m}}(T) \cap \mathfrak{q}^{n} T=0$ for all $n \geq n_{0}$. This gives the relations

$$
\begin{aligned}
\operatorname{length}_{A}\left(T / \mathfrak{q}^{n} T\right) & =\operatorname{length}_{A}\left(\bar{T} / \mathfrak{q}^{n} \bar{T}\right)+\operatorname{length}_{A}\left(\Gamma_{\mathfrak{m}}(T)\right) \text { for all } n \geq n_{0} \\
P_{T, \mathfrak{q}}(\mathbf{x}) & =P_{\bar{T}, \mathfrak{q}}(\mathbf{x})+\operatorname{length}_{A}\left(\Gamma_{\mathfrak{m}}(T)\right) \\
e_{0}(\mathfrak{q}, T) & =e_{0}(\mathfrak{q}, \bar{T}) \\
e_{1}(\mathfrak{q}, T) & =e_{1}(\mathfrak{q}, \bar{T})-\operatorname{length}_{A}\left(\Gamma_{\mathfrak{m}}(T)\right)
\end{aligned}
$$

2.2. Notation. A) Let

$$
\mathcal{R}(\mathfrak{q}):=\bigoplus_{n \geq 0} \mathfrak{q}^{n}, \quad \mathcal{R}(\mathfrak{q}, T):=\bigoplus_{n \geq 0} \mathfrak{q}^{n} T
$$

denote the Rees ring of $\mathfrak{q}$ and the Rees module of $T$ with respect to $\mathfrak{q}$, respectively.

B) In addition, let

$$
\begin{aligned}
\operatorname{Gr}(\mathfrak{q}) & :=\mathcal{R}(\mathfrak{q}) / \mathfrak{q} \mathcal{R}(\mathfrak{q})=\bigoplus_{n \geq 0} \mathfrak{q}^{n} / \mathfrak{q}^{n+1} \\
\operatorname{Gr}(\mathfrak{q}, T) & :=\mathcal{R}(\mathfrak{q}, T) / \mathfrak{q} \mathcal{R}(\mathfrak{q}, T)=\bigoplus_{n \geq 0} \mathfrak{q}^{n} T / \mathfrak{q}^{n+1} T
\end{aligned}
$$

denote the associated graded ring of $\mathfrak{q}$ and the associated graded module of $T$ with respect to $\mathfrak{q}$, respectively.

2.3. Notation. For a finitely generated graded module $M$ over the Noetherian homogeneous ring $R=\bigoplus_{n \geq 0} R_{n}$ let reg( $\left.M\right)$ denote the (Castelnuovo-Mumford) regularity of $M$, thus

$$
\operatorname{reg}(M):=\inf \left\{r \in \mathbb{Z} \mid H_{R_{+}}^{i}(M)_{n-i+1}=0, \forall i \in \mathbb{N}_{0}, \forall n \geq r\right\} .
$$

2.4. Lemma. Let $\operatorname{depth}_{A}(T) \neq 0$. Then $\operatorname{reg}(\operatorname{Gr}(\mathfrak{q}, T)) \leq \operatorname{reg}(\operatorname{Gr}(\mathfrak{q}))$. 
Proof. Let $\mathbf{x}$ be an indeterminate. Then $B:=A[\mathbf{x}]_{\mathfrak{m} A[\mathbf{x}]}$ is a Noetherian local flat one-dimensional extension ring of $A$ with maximal ideal $\mathfrak{n}:=\mathfrak{m} B$ and infinite residue field $B / \mathfrak{n}$. Moreover $\mathfrak{q} B \subseteq B$ is an $\mathfrak{n}$-primary ideal and $T \otimes_{A} B$ is a finitely generated $B$-module of dimension 1 and depth $\neq 0$.

In addition, there is an isomorphism of graded rings $\operatorname{Gr}(\mathfrak{q} B) \cong \operatorname{Gr}(\mathfrak{q}) \otimes_{A} B$ and an isomorphism of graded $\operatorname{Gr}(\mathfrak{q} B)$-modules $\operatorname{Gr}\left(\mathfrak{q} B, T \otimes_{A} B\right) \cong \operatorname{Gr}(\mathfrak{q}, T) \otimes_{A} B$. In view of the graded flat base-change property of local cohomology (cf. [B-S]), these latter isomorphisms induce

$$
\operatorname{reg}(\operatorname{Gr}(\mathfrak{q} B))=\operatorname{reg}(\operatorname{Gr}(\mathfrak{q}))
$$

and

$$
\operatorname{reg}\left(\operatorname{Gr}\left(\mathfrak{q} B, T \otimes_{A} B\right)\right)=\operatorname{reg}(\operatorname{Gr}(\mathfrak{q}, T)) .
$$

Altogether, we thus may replace $(A, \mathfrak{m}), \mathfrak{q}$ and $T$ respectively by $(B, \mathfrak{n}), \mathfrak{q} B$ and $T \otimes_{A} B$ and hence assume that $A / \mathfrak{m}$ is infinite.

As $\mathfrak{q}$ is $\mathfrak{m}$-primary, its analytic spread equals $\operatorname{dim}(A)=1$. So there is some $x \in \mathfrak{q}$ such that $A x$ is a minimal reduction of $\mathfrak{q}$. As $A / \mathfrak{m}$ is infinite the reduction number of $\mathfrak{q}$ with respect to $A x$ is $\leq \operatorname{reg}(\operatorname{Gr}(\mathfrak{q})$ ) (cf. [T], Proposition 3.2 ] or [B-S 18.3.12]) so that $x \mathfrak{q}^{n}=\mathfrak{q}^{n+1}$ for all $n \geq \operatorname{reg}(\operatorname{Gr}(\mathfrak{q}))$. Let $x^{*}=$ $(0, x, 0, \cdots) \in \mathcal{R}(\mathfrak{q})_{1}$ be the element $x \in \mathfrak{q}$ considered as a one-form in $\mathcal{R}(\mathfrak{q})$. It follows that $\sqrt{\mathcal{R}(\mathfrak{q})_{+}}=\sqrt{x^{*} \mathcal{R}(\mathfrak{q})}$ so that $H_{\mathcal{R}(\mathfrak{q})_{+}}(\mathcal{R}(\mathfrak{q}, T))=H_{\left(x^{*}\right)}^{i}(\mathcal{R}(\mathfrak{q}, T))$ for all $i \geq 0$. Therefore $H_{\mathcal{R}(\mathfrak{q})_{+}}^{i}(\mathcal{R}(\mathfrak{q}, T))=0$ for all $i>1$. As $x \in A$ is a parameter, $\operatorname{depth}_{A}(T)=1=\operatorname{dim}(A)$ implies that $x$ is $T$-regular. So $x^{*}$ is $\mathcal{R}(\mathfrak{q}, T)$-regular and hence $H_{\mathcal{R}(\mathfrak{q})_{+}}^{0}(\mathcal{R}(\mathfrak{q}, T))=0$. Moreover we have the following short exact sequence of graded $\mathcal{R}(\mathfrak{q})$-modules (cf. [B-S, 2.2.17]):

$$
0 \rightarrow \mathcal{R}(\mathfrak{q}, T) \stackrel{\eta}{\longrightarrow} \mathcal{R}(\mathfrak{q}, T)_{x^{*}} \rightarrow H_{\left(x^{*}\right)}^{1}(\mathcal{R}(\mathfrak{q}, T)) \rightarrow 0 .
$$

As $x^{*} \mathcal{R}(\mathfrak{q}, T)_{n}=x \mathfrak{q}^{n} T=\mathfrak{q}^{n+1} T=\mathcal{R}(\mathfrak{q}, T)_{n+1}$ for all $n \geq \operatorname{reg}(\operatorname{Gr}(\mathfrak{q}))$, the natural map $\eta$ becomes an isomorphism in all degrees $\geq \operatorname{reg}(\operatorname{Gr}(\mathfrak{q}))$ so that

$$
H_{\mathcal{R}(\mathfrak{q})_{+}}^{1}(\mathcal{R}(\mathfrak{q}, T))_{n}=H_{\left(x^{*}\right)}^{1}(\mathcal{R}(\mathfrak{q}, T))_{n}=0
$$

for all $n \geq \operatorname{reg}(\operatorname{Gr}(\mathfrak{q}))$. Thus, finally we get $\operatorname{reg}(\mathcal{R}(\mathfrak{q}, T)) \leq \operatorname{reg}(\operatorname{Gr}(\mathfrak{q}))$.

Now, in view of the well-known behaviour of regularities in short exact sequences (cf. [B-S, 15.2.15]) and as $\operatorname{reg}(\mathcal{R}(\mathfrak{q}, T)) \geq 0$ (cf. [B-S, 15.3.1]), we get our claim by the graded exact sequences

$$
\begin{aligned}
& 0 \rightarrow \mathfrak{q} \mathcal{R}(\mathfrak{q}, T) \rightarrow \mathcal{R}(\mathfrak{q}, T)(1) \rightarrow[T]_{-1} \rightarrow 0 \\
& 0 \rightarrow \mathfrak{q} \mathcal{R}(\mathfrak{q}, T) \rightarrow \mathcal{R}(\mathfrak{q}, T) \rightarrow \operatorname{Gr}(\mathfrak{q}, T) \rightarrow 0
\end{aligned}
$$

2.5. Lemma. Let $r \geq \operatorname{reg}(\operatorname{Gr}(\mathfrak{q}))$, let $n \geq r$ and assume that $\operatorname{depth}_{A}(T)=1$. Then

$$
\operatorname{length}_{A}\left(T / \mathfrak{q}^{n+1} T\right)=e_{0}(\mathfrak{q}, T) n-r e_{0}(\mathfrak{q}, T)+\operatorname{length}_{A}\left(T / \mathfrak{q}^{r+1} T\right) .
$$

Proof. For all $n \in \mathbb{N}_{0}$ we have

$$
\operatorname{length}_{A / \mathfrak{q}}\left(\operatorname{Gr}(\mathfrak{q}, T)_{n}\right)=\operatorname{length}_{A}\left(T / \mathfrak{q}^{n+1} T\right)-\operatorname{length}_{A}\left(T / \mathfrak{q}^{n} T\right) .
$$

For all $n \gg 0$, the right-hand side of this equality takes the value $P_{T, \mathfrak{q}}(n)-$ $P_{T, \mathfrak{q}}(n-1)=e_{0}(\mathfrak{q}, T)$, so that the characteristic function of the graded $\operatorname{Gr}(\mathfrak{q})$ module $\operatorname{Gr}(\mathfrak{q}, T)$ takes the constant value $e_{0}(\mathfrak{q}, T)$. As $\operatorname{reg}(\operatorname{Gr}(\mathfrak{q}, T)) \leq r$ it follows 
that length $\tan _{A / \mathfrak{q}}\left(\operatorname{Gr}(\mathfrak{q}, T)_{n}\right)=e_{0}(\mathfrak{q}, T)$ for all $n>r$ and hence $\operatorname{length}_{A}\left(T / \mathfrak{q}^{n+1} T\right)-$ $\operatorname{length}_{A}\left(T / \mathfrak{q}^{n} T\right)=e_{0}(\mathfrak{q}, T)$ for all $n \geq r+1$. So, for all $n \geq r$ we get

$$
\begin{aligned}
\operatorname{length}_{A}\left(T / \mathfrak{q}^{n+1} T\right) & =\sum_{m=r+1}^{n}\left(\operatorname{length}_{A}\left(T / \mathfrak{q}^{m+1} T\right)-\operatorname{length}_{A}\left(T / \mathfrak{q}^{m} T\right)\right) \\
& +\operatorname{length}_{A}\left(T / \mathfrak{q}^{r+1} T\right)=e_{0}(\mathfrak{q}, T)(n-r)+\operatorname{length}_{A}\left(T / \mathfrak{q}^{r+1} T\right) \\
& =e_{0}(\mathfrak{q}, T) n-r e_{0}(\mathfrak{q}, T)+\operatorname{length}_{A}\left(T / \mathfrak{q}^{r+1} T\right)
\end{aligned}
$$

2.6. Proposition. Let $r \geq \operatorname{reg}(\operatorname{Gr}(\mathfrak{q}))$. Then

$$
e_{1}(\mathfrak{q}, T)=(r+1) e_{0}(\mathfrak{q}, T)-\operatorname{length}_{A}\left(T / \mathfrak{q}^{r+1} T\right)-\operatorname{length}_{A}\left(\mathfrak{q}^{r+1} T \cap \Gamma_{\mathfrak{m}}(T)\right) .
$$

Proof. As $\bar{T}$ has dimension and depth 1, Lemma2.5 gives $e_{1}(\mathfrak{q}, \bar{T})=(r+1) e_{0}(\mathfrak{q}, \bar{T})-$ length $_{A}\left(\bar{T} / \mathfrak{q}^{r+1} \bar{T}\right)$. As

$$
e_{0}(\mathfrak{q}, T)=e_{0}(\mathfrak{q}, \bar{T}), e_{1}(\mathfrak{q}, T)=e_{1}(\mathfrak{q}, \bar{T})-\operatorname{length}_{A}\left(\Gamma_{\mathfrak{m}}(T)\right)
$$

and

$$
\begin{aligned}
\operatorname{length}_{A}\left(\bar{T} / \mathfrak{q}^{r+1} \bar{T}\right)= & \operatorname{length}_{A}\left(T / \mathfrak{q}^{r+1} T\right)-\operatorname{length}_{A}\left(\Gamma_{\mathfrak{m}}(T)\right) \\
& +\operatorname{length}_{A}\left(\Gamma_{\mathfrak{m}}(T) \cap \mathfrak{q}^{r+1} T\right),
\end{aligned}
$$

(cf. 2.1 C)), we get our claim.

\section{Antipolynomial growth of first Hilbert-Samuel coefficients AND BOUNDEDNESS OF POSTULATION NUMBERS}

Now, we are ready to formulate and to prove our first main result.

3.1. Theorem. Let $R=\bigoplus_{n>0} R_{n}$ be a Noetherian homogeneous ring such that $\left(R_{0}, \mathfrak{m}_{0}\right)$ is local and of dimension 1 . Let $\mathfrak{q}_{0} \subseteq R_{0}$ be an $\mathfrak{m}_{0}$-primary ideal, let $M$ be a finitely generated graded $R$-module and let $i \in \mathbb{N}_{0}$. Assume that $\operatorname{dim}_{R_{0}}\left(H_{R_{+}}^{i}(M)_{n}\right)$ $=1$ for all $n \ll 0$. Then there is a polynomial $S(\mathbf{x}) \in \mathbb{Q}[\mathbf{x}]$ of degree $<i$ such that

$$
e_{1}\left(\mathfrak{q}_{0}, H_{R_{+}}^{i}(M)_{n}\right)=S(n) \text { for all } n \ll 0 .
$$

Proof. By our hypotheses there is some $r \geq \operatorname{reg}\left(\operatorname{Gr}\left(\mathfrak{q}_{0}\right)\right)$ such that $\operatorname{dim}_{R_{0}}\left(H_{R_{+}}^{i}(M)_{n}\right)$ $=1$ for all $n \leq-r$. So by Proposition 2.6 we obtain for all $n \leq-r$ :

$$
\begin{aligned}
& e_{1}\left(\mathfrak{q}_{0}, H_{R_{+}}^{i}(M)_{n}\right)=(r+1) e_{0}\left(\mathfrak{q}_{0}, H_{R_{+}}^{i}(M)_{n}\right) \\
& -\operatorname{length}_{R_{0}}\left(H_{R_{+}}^{i}(M)_{n} / \mathfrak{q}_{0}^{r+1} H_{R_{+}}^{i}(M)_{n}\right) \\
& -\operatorname{length}_{R_{0}}\left(\mathfrak{q}_{0}^{r+1} H_{R_{+}}^{i}(M)_{n} \cap \Gamma_{\mathfrak{m}_{0}}\left(H_{R_{+}}^{i}(M)_{n}\right)\right) .
\end{aligned}
$$

According to (1.1) there are polynomials $P, Q \in \mathbb{Q}[\mathbf{x}]$ of degree $<i$ such that

$$
e_{0}\left(\mathfrak{q}_{0}, H_{R_{+}}^{i}(M)_{n}\right)=Q(n), \text { length } R_{0}\left(H_{R_{+}}^{i}(M)_{n} / \mathfrak{q}_{0}^{r+1} H_{R_{+}}^{i}(M)_{n}\right)=P(n)
$$

for all $n \ll 0$. 
By [B-F-T, Theorem 2.5], the graded $R$-module $\Gamma_{\mathfrak{m}_{0} R}\left(H_{R_{+}}^{i}(M)\right)$ is Artinian, and hence so is its graded submodule

$$
U:=\mathfrak{q}_{0}^{r+1} H_{R_{+}}^{i}(M) \cap \Gamma_{\mathfrak{m}_{0} R}\left(H_{R_{+}}^{i}(M)\right) .
$$

So, there is a polynomial $F \in \mathbb{Q}[\mathbf{x}]$ such that length $_{R_{0}}\left(U_{n}\right)=F(n)$ for all $n \ll 0$ (cf. [Ki]). According to (1.1) there is a polynomial $\bar{Q} \in \mathbb{Q}[\mathbf{x}]$ of degree $<i$ such that length $R_{0}\left(\Gamma_{\mathfrak{m}_{0}}\left(H_{R_{+}}^{i}(M)_{n}\right)\right)=\bar{Q}(n)$ for all $n \ll 0$. As $U_{n} \subseteq \Gamma_{\mathfrak{m}_{0}}\left(H_{R_{+}}^{i}(M)_{n}\right)$ for all $n$, it follows that $\operatorname{deg}(F) \leq \operatorname{deg}(\bar{Q})<i$.

As $U_{n}=\mathfrak{q}_{0}^{r+1} H_{R_{+}}^{i}(M)_{n} \cap \Gamma_{\mathfrak{m}_{0}}\left(H_{R_{+}}^{i}(M)_{n}\right)$, the equality given at the beginning of this proof yields

$$
e_{1}\left(\mathfrak{q}_{0}, H_{R_{+}}^{i}(M)_{n}\right)=(r+1) Q(n)-P(n)-F(n) \text { for all } n \ll 0 .
$$

This proves our claim.

Now we prove the announced boundedness of postulation numbers (cf. (1.3)). We begin with a preliminary remark.

3.2. Remark. Let $R=\bigoplus_{n \in \mathbb{Z}} R_{n}$ be a graded ring, let $W=\bigoplus_{n \in \mathbb{Z}} W_{n}$ be a graded and Artinian $R$-module and let $\left(U^{(m)}\right)_{m \in \mathbb{N}_{0}}$ be a descending sequence of graded submodules of $W$. Assume that for each $n \in \mathbb{Z}$ there is some $m_{n} \in \mathbb{N}_{0}$ such that $U_{n}^{\left(m_{n}\right)}=0$.

As $W$ is Artinian, there is some $t \in \mathbb{N}_{0}$ such that $U^{(m)}=U^{(t)}$ for all $m \geq t$. So, for each $n \in \mathbb{Z}$ and for each $m \geq \max \left\{t, m_{n}\right\}$, we obtain $U_{n}^{(t)}=U_{n}^{(m)} \subseteq U_{n}^{\left(m_{n}\right)}=0$. Therefore $U^{(t)}=0$.

3.3. Theorem. Let $R=\bigoplus_{n \geq 0} R_{n}$ be a Noetherian homogeneous ring such that $\left(R_{0}, \mathfrak{m}_{0}\right)$ is local and of dimension $\leq 1$. Let $\mathfrak{q}_{0} \subseteq R_{0}$ be an $\mathfrak{m}_{0}$-primary ideal, let $M$ be a finitely generated graded $R$-module and let $i \in \mathbb{N}_{0}$. Then, there is some $c \in \mathbb{N}_{0}$ such that $\mu\left(\mathfrak{q}_{0}, H_{R_{+}}^{i}(M)_{n}\right) \leq c$ for all $n \in \mathbb{Z}$.

Proof. By $[$ B-F-T Theorem 3.5 e)] there is some $\delta \in\{0, \pm 1\}$ such that

$$
\operatorname{dim}_{R_{0}}\left(H_{R_{+}}^{i}(M)_{n}\right)=\delta \text { for all } n \ll 0 .
$$

If $\delta=-1$ we have $H_{R_{+}}^{i}(M)_{n}=0$ for all but finitely many values of $n$ and our claim is clear.

If $\delta \geq 0$ we shall apply Remark 3.2 to the graded Artinian $R$-module

$$
W:=\Gamma_{\mathfrak{m}_{0} R}\left(H_{R_{+}}^{i}(M)\right)
$$

(cf. B-F-T, Theorem $2.5 \mathrm{~b}$ )]). First, let $\delta=0$, so that $W_{n}=H_{R_{+}}^{i}(M)_{n}$ for all $n \ll 0$. If we apply Remark 3.2 with $U^{(m)}:=\mathfrak{q}_{0}^{m} W$, we find some $t \in \mathbb{N}_{0}$ with $\mathfrak{q}_{0}^{t} W=0$ so that $\mathfrak{q}_{0}^{t} H_{R_{+}}^{i}(M)_{n}=0$ for all $n \ll 0$. As $H_{R_{+}}^{i}(M)_{n}=0$ for all $n \gg 0$ we get our claim if $\delta=0$.

So, let $\delta=1$. If we apply Remark 3.2 with $U^{(m)}:=W \cap \mathfrak{q}_{0}^{m} H_{R_{+}}^{i}(M)$ and use the lemma of Artin-Rees we find some $t \in \mathbb{N}$ with $\Gamma_{\mathfrak{m}_{0} R}\left(H_{R_{+}}^{i}(M)\right) \cap \mathfrak{q}_{0}^{t} H_{R_{+}}^{i}(M)=0$, so that $\Gamma_{\mathfrak{m}_{0}}\left(H_{R_{+}}^{i}(M)_{n}\right) \cap \mathfrak{q}_{0}^{t} H_{R_{+}}^{i}(M)_{n}=0$ for all $n \in \mathbb{Z}$. Now, let $n_{0}$ be such that $\operatorname{dim}_{R_{0}}\left(H_{R_{+}}^{i}(M)_{n}\right)=1$ for all $n \leq n_{0}$. Then, for each $r \geq \max \left\{\operatorname{reg}\left(\operatorname{Gr}\left(\mathfrak{q}_{0}\right)\right), t-1\right\}$ 
and each $n \leq n_{0}$, Proposition [2.6 - applied with $A=R_{0}, \mathfrak{q}=\mathfrak{q}_{0}$ and $T=$ $H_{R_{+}}^{i}(M)_{n}$ - gives

$$
\begin{aligned}
& \operatorname{length}_{R_{0}}\left(H_{R_{+}}^{i}(M)_{n} / \mathfrak{q}_{0}^{r+1} H_{R_{+}}^{i}(M)_{n}\right) \\
& \quad=(r+1) e_{0}\left(\mathfrak{q}_{0}, H_{R_{+}}^{i}(M)_{n}\right)-e_{1}\left(\mathfrak{q}_{0}, H_{R_{+}}^{i}(M)_{n}\right)=P_{H_{R_{+}}^{i}(M)_{n}, \mathfrak{q}_{0}}(r) .
\end{aligned}
$$

As $H_{R_{+}}^{i}(M)_{n}=0$ for all $n \gg 0$, this proves our claim.

3.4. Corollary. Let $R=\bigoplus_{n>0} R_{n}$ be a Noetherian homogeneous ring such that $\left(R_{0}, \mathfrak{m}_{0}\right)$ is local and of dimension $\leq 1$. Let $\mathfrak{q}_{0} \subseteq R_{0}$ be an $\mathfrak{m}_{0}$-primary ideal, let $M$ be a finitely generated graded $R$-module and let $i \in \mathbb{N}_{0}$. Then, there are integers $n_{0} \in \mathbb{Z}, c \in \mathbb{N}_{0}$ and polynomials $S, Q \in \mathbb{Q}[\mathbf{x}]$ of degree $<i$ such that for each $n \leq n_{0}$ and each $r \geq c$ we have

$$
\text { length }_{R_{0}}\left(H_{R_{+}}^{i}(M)_{n} / \mathfrak{q}_{0}^{r+1} H_{R_{+}}^{i}(M)_{n}\right)=Q(n)(r+1)-S(n) .
$$

Proof. Immediate by Theorem 3.1. Theorem 3.3 and B-F-T, Theorem 3.5].

\section{REFERENCES}

[B-F-L] M. BRODMANN, S. FUMASOLI and C.S. LIM: Low-codimensional associated primes of graded components of local cohomology modules, Journal of Algebra 275 (2004), 867-882. MR2052643

[B-F-T] M. BRODMANN, S. FUMASOLI and R. TAJAROD: Local cohomology over homogeneous rings with one-dimensional local base ring, Proceedings of the AMS 131, No 10 (2003), 2977-2985. MR1993202 (2004f:13021)

[B-H] M. BRODMANN and M. HELLUS: Cohomological patterns of coherent sheaves over projective schemes, Journal of Pure and Applied Algebra 172 (2002), 165-182. MR 1906872 (2003f:13017)

[B-K-S] M. BRODMANN, M. KATZMAN and R.Y. SHARP: Associated primes of graded components of local cohomology modules, Transactions of the AMS 354, No 11 (2002), 42614283. MR1926875 (2003h:13020)

[B-S] M. BRODMANN and R.Y. SHARP: Local cohomology: an algebraic introduction with geometric applications, Cambridge Studies in Advanced Mathematics 60, Cambridge University Press (1998). MR1613627|(99h:13020)

[E] D. EISENBUD: Commutative algebra with a view towards algebraic geometry, Springer, New York (1996). MR1322960 (97a:13001)

[K] M. KATZMAN: An example of an infinite set of associated primes of a local cohomology module, Journal of Algebra 252 (2002), 161-166. MR1922391(2003h:13021)

[Ki] D. KIRBY: Artinian modules and Hilbert polynomials, Quarterly Journal of Mathematics, Oxford (2) 24 (1973), 47-57. MR.0316446 (47:4993)

[L] C.S. LIM: Graded local cohomology modules and their associated primes, Communications in Algebra 32, No 2 (2004), 727-745.

[S] A.K. SINGH: p-torsion elements in local cohomology modules, Mathematics Research Letters 7 (2000), 165-176. MR:1764314|(2001g:13039)

[T] N.V. TRUNG: Reduction exponent and degree bound for the defining equations of graded rings, Proceedings of the AMS 101 (1987), 229 -236. MR.0902533 (89i:13031)

Institute of Mathematics, University of ZÜRICH, Winterthurerstrasse 190, CH-8057 Zürich, SWITZERLAND

E-mail address: brodmann@math.unizh.ch

Institute of Mathematics, University of ZÜRICh, Winterthurerstrasse 190, CH-8057 Zürich, SWITZERLAND

E-mail address: fred@math.unizh.ch 